\title{
Control de costos \\ DE PRODUCCIÓN EN \\ ORGANIZACIONES HOTELERAS
}

\section{Control of production COSTS IN HOTEL ORGANIZATIONS}

\author{
Mónica Patricia Mayorga Díaz ${ }^{1}$ \\ Edith Marianela Fernández Agreda ${ }^{2}$
}

1 Doctora en ciencias económicas. Docente investigadora. Facultad de Sistemas Mercantiles de la Universidad Regional Autónoma de Los Andes. Ambato, Ecuador. Correo electrónico: monikmayorgad@gmail.com

Orcid: https://orcid.org/00000003-3071-236X

2 Ingeniera en Contabilidad y Auditoría. Profesional independiente. Ambato, Ecuador. Correo electrónico: edithcita_1524@hotmail.com

Orcid: https://orcid.org/ 0000-0002-2188-7427

Código JeL: D20

Fecha de recepción: 16/10/2019

Fecha de aceptación: 10/04/2020

DOI: https://doi.org/10.18601/16577175.n27.07 


\section{Resumen}

En el contexto empresarial moderno se encuentra que la dinámica por la que ha adoptado el sector hotelero constituye la obtención de rentabilidad con baja inversión en activos de larga duración, y en muchos de los casos inclusive se realiza el registro de costos de producción de forma empírica, convirtiéndose en prioridad ofrecer alojamiento digno sin considerar las expectativas del consumidor (turista). En el Ecuador las organizaciones hoteleras han experimentado un crecimiento constante, por lo que el objetivo de esta investigación consiste en analizar el control de costos de procesos de producción, optimización de recursos y acciones estratégicas, que permitan el mejoramiento de la administración económica en las organizaciones hoteleras, para esto es necesario evaluar la situación financiera y el control de costos en estas empresas, mediante un análisis categorial y revisión documental de fuentes secundarias que permitan identificar la fragmentación de la bibliografía y el estado de la práctica.

Palabras clave: herramientas; objetivos; optimizar recursos; rendimiento; rentabilidad.

\section{Abstract}

In the modern business context, it is observed that the dynamics by which the hotel sector has adopted constitutes the obtaining of profitability with low investment in long-term assets, and in many cases they even register production costs empirically, becoming a priority to offer decent accommodation without considering the expectations of the consumer (tourist). In Ecuador hotel organizations have experienced a constant growth, so the objective of this research is to analyze the cost control of production processes, optimization of resources and strategic actions, which allow the improvement of the economic administration in hotel organizations For this, it is necessary to assess the financial situation and cost control in these companies, through a categorical analysis and documentary review of secondary sources that identify the fragmentation of the bibliography and the state of practice.

Keywords: objectives; tools; optimize resources; performance; profitability.

\section{INTRODUCCIÓN}

En la antigüedad los hoteles desempeñaron un papel destacado para el desarrollo y aumento de las naciones, en la Edad Media los negociantes que debían viajar grandes extensiones de kilómetros en busca de mercadería requerían sitios con servicios donde pasar sus noches y así fueron extendiéndose los hospedajes en casas de hogares de familias, posadas, hosterías, hoteles. Sus inicios en Ecuador surgen en el año 1955, cuando un grupo de empresarios hoteleros guayaquileños decidió constituir y fundar 
una organización nacional que aglomerara y simbolizara los intereses y utilidades del sector hotelero. En la provincia de Tungurahua encontramos una gran variedad de hoteles de 4, 3, 2 y 1 estrellas, al igual que hostales, hosterías y residenciales en los diferentes cantones de la misma, en donde los turistas pueden conocer varios sectores culturales y naturales, saborear exquisitos platos típicos y adquirir llamativas artesanías de nuestros artesanos. En el cantón Ambato contamos con una diversidad de alojamientos como hoteles, residenciales, hosterías y hostales, los cuales ofrecen distintos servicios para los turistas nacionales y extranjeros que visitan esta ciudad, tales como el servicio de hospedaje, restaurante, garaje, lavandería, camarería, transporte, guianza, discoteca, bar, piscina, entre otros, por un precio establecido por cada hotel y por cada categoría que brindan estas instituciones (Rodríguez, 2018).

El costo de producción es un conjunto de gastos que son necesarios para fabricar un bien o servicio que ayuda a manejar y regular el movimiento de los diferentes materiales mientras se realiza un ciclo de elaboración, que parte desde la incautación de las materias primas hasta la transferencia del producto ya finalizado, es la forma de manejar y regular el movimiento de los diferentes materiales mientras se realiza un ciclo de elaboración, para la entrega de un producto terminado.

En el caso del sistema hotelero se calculan los costos de producción de forma directa e indirecta. Un costo directo es aquel que puede identificarse directamente con un proceso, producto, trabajo o servicio. Como ejemplo de costos directos puede citarse el costo de los comestibles que se consumen en un restaurante o el salario del cantinero de un bar. Un costo indirecto es aquel que no puede atribuirse directamente a una producción o servicio, como por ejemplo el salario del director del hotel o la depreciación del edificio; los costos indirectos se pueden distribuir a las producciones, servicios o puntos de venta del hotel de acuerdo con una base o índice que refleje la manera en que se supone que se utilizan o aplican esos elementos indirectos en las producciones o servicios a los que se distribuye. Pero las bases de distribución de los costos indirectos son generalmente arbitrarias o se fundamentan en bases teóricas o cuestiones de criterios, por lo que actualmente la mayoría de las entidades rechaza la distribución de los costos indirectos y los registran como tales por su naturaleza (Rodríguez, 2017). En tal sentido, es necesario tratar las aristas que involucran el objetivo de esta investigación.

La gestión de costos constituye procedimientos que permiten optimizar recursos, acciones estratégicas con la finalidad de alcanzar objetivos y administrar sus recursos financieros evaluando la comprensión de costos y la contribución para el mejoramiento de la administración económica de las organizaciones siendo una de las herramientas más importantes para tomar decisiones por el cual las organizaciones informan y controlan el negocio de la empresa de una manera eficiente; es decir, la organización tiene la facilidad y los mecanismos para identificar y conocer la situación actual financiera hotelera de la gestión de costos con el objetivo de diagnosticar el acontecimiento que puede ocurrir a futuro, determinando de manera competente los costos de reducción que garantizan el éxito y el control de 
las acciones (Cárdenas, 2016); (Ediciones, 2016); (García, 2016); (Liendo, 2016); (González, 2016); (Gómez, 2018).

Así como también integra el eje de crecimiento, productividad y competitividad de las organizaciones hoteleras del nuevo siglo dirigido a la generalización de negocios y postura en los mercados, considerándose uno de los instrumentos más utilizados para la dirección y la toma de decisiones de la institución y en cada una de sus ámbitos a través de la implementación de sistemas de costos, indicadores financieros, control interno, presupuestos, entre otras actividades que coadyuven al logro de los objetivos planificados de las empresas (Pereira, 2019); (Roque, 2019).

A partir de la bibliografía consultada se concluye que la gestión de costos constituye una herramienta que permite el crecimiento y la productividad en cualquier tipo de organización, sin embargo, no puede estar independiente de los resultados de la evaluación financiera, por lo que es necesario tratar esta arista de investigación.

La evaluación financiera permite verificar el rendimiento futuro de objetivos llegando a detectar dificultades venideras de la situación económica actual de la empresa con la finalidad de establecer la toma de decisiones que tendrá la institución, para ello es necesario realizar la medición de la evaluación financiera mediante el cálculo de los análisis financieros, estados financieros e indicadores económicos.

Es importante la evaluación financiera en las instituciones ya que es una guía que orienta a los hoteles en el área de la obtención de rendimiento a futuro que sirve para analizar sus ingresos sus gastos, de una manera adecuada y acertada con la finalidad de alcanzar objetivos para tomar decisiones por parte de los directivos de la organización mediante procesos para identificar los beneficios y costos de la organización (Villanueva, 2015); (Serrano, 2016); (Aponte, 2017); (Mayenberger, 2017).

$\mathrm{Su}$ aplicación es fundamental para informar y guiar en sus decisiones a la alta gerencia con base en razones y flujos de fondos que pueda implementarlas en su proceso de decisión y planeación estratégica logrando optimizar los resultados esperados obteniendo como objetivo tomar decisiones sobre cuándo y cuánto invertir o desinvertir, es preferible disponer de más información que de menos, ya que ello le puede ayudar a reducir la incertidumbre relacionada con la rentabilidad y riesgo futuros (Católico, 2019); (Calvo, 2010).

Los elementos de la gestión de costos se basan en tres principales componentes: (1) materia prima, (2) mano de obra directa, (3) costos indirectos de fabricación o producción; estos elementos de gestión se caracterizan por su rentabilidad, competitividad, punto de equilibrio, recursos humanos, materiales para actividades y equipos con el fin de obtener una correcta asignación de costos, y cuanto más directa sea ésta, dará como resultado un mejor costo de la producción (Jiménez, 2016); (Otálora, 2016); (Aspiolea, 2017); (Rodríguez, 2018).

Por consiguiente, una herramienta de gestión de costos genera información relevante para la toma de decisiones en mejorar aspectos económicos, convirtiéndose en una eficaz herramienta de gestión, mediante las actividades desarrolladas dentro de una empresa basándose en indicadores financieros, control interno, presupuestos, entre otras acciones que colaboran al logro de los objetivos estratégicos de la organización 
(Roque, 2019). Una vez determinada la fragmentación en la bibliografía es menester analizar el marco jurídico ecuatoriano vigente, en relación al tema de estudio (tabla 1).

Tabla 1. Marco jurídico

\begin{tabular}{|c|c|}
\hline Marco jurídico vigente & Artículo \\
\hline $\begin{array}{l}\text { Ley de Régimen Tributario } \\
\text { Interno (LORTI) } \\
\text { Registro Oficial } 145 \text { del } \\
17 \text { de diciembre de } 2013\end{array}$ & $\begin{array}{l}\text { Art. 13. Presentar al Servicio de Rentas Internas, los correspondien- } \\
\text { tes estados de resultados y en caso de existir proceso productivo, el } \\
\text { estado de costos de producción, en donde se establezcan claramente } \\
\text { los valores de ingresos, costos y gastos y utilidades atribuibles de } \\
\text { manera directa a la inversión nueva y productiva (Asamblea Nacio- } \\
\text { nal, 2013, art.13). }\end{array}$ \\
\hline $\begin{array}{l}\text { Código Orgánico de la Producción, } \\
\text { Comercio e Inversiones } \\
\text { Registro Oficial Suplemento } \\
\text { n. }{ }^{\circ} 351,29 \text { de diciembre de } 2010\end{array}$ & $\begin{array}{l}\text { Art. 62. Acceso a la banca pública. El Consejo Sectorial de la Polí- } \\
\text { tica Económica determinará y vigilará el acceso de todos los actores } \\
\text { productivos al financiamiento de la entidad hotelero; establecerá } \\
\text { los lineamientos e incentivos la producción y procurar la reducción } \\
\text { de los costos de intermediación financiera (Asamblea Nacional, } \\
2010 \text {, art. 62). }\end{array}$ \\
\hline $\begin{array}{c}\text { Ley de Turismo } \\
\text { Registro Oficial } 244 \\
\text { del } 5 \text { de enero de } 2004 \text { - } \\
22 \text { de enero de } 2015\end{array}$ & $\begin{array}{l}\text { Art. 23. Tasas por servicios técnicos y administrativos. De conformi- } \\
\text { dad con lo que dispone el art. 17-A de la Ley de Modernización del } \\
\text { Estado, el Ministerio de Turismo, a través de un acuerdo ministerial } \\
\text { y los organismos seccionales autónomos a quien se ha descentra- } \\
\text { lizado la competencia podrán establecer el pago de tasas por los } \\
\text { servicios de control (Constitución de la República, 2015, art. 23). }\end{array}$ \\
\hline $\begin{array}{l}\text { Reglamento de alojamiento } \\
\text { Registro Oficial Suplemento } 465 \\
\text { del } 24 \text { de marzo de } 2015 \text { - } \\
18 \text { de febrero de } 2016\end{array}$ & $\begin{array}{l}\text { Art 3. Los hoteles son conocidos por las siguientes características: } \\
\text { "[...] cuarto de bańo y aseo, cerradura para puerta de acceso a la } \\
\text { habitación, almohada Frigo bar, portamaletas, clóset y/o armario, } \\
\text { escritorio y/o mesa. silla, sillón o sofá, funda de lavandería, luz de } \\
\text { velador o cabecera por plaza, cortina completa y visillo o blackout } \\
\text { y visillo, el blackout o la cortina completa pueden ser sustituidos } \\
\text { por puerta interior de la ventana, cortinas o persianas. Televisión } \\
\text { ubicada en mueble o soporte con acceso a canales nacionales e } \\
\text { internacionales, con televisión por cable o televisión satelital con } \\
\text { acceso a canales nacionales, teléfono en habitación [...]" (Ministerio } \\
\text { de Turismo, 2016, art. 3). } \\
\text { Art 12. Los servicios de los hoteles que ofrecen en el Ecuador son: } \\
\text { prestación de hospedaje en apartamentos que integren una unidad } \\
\text { para este uso exclusivo. Cada apartamento debe estar compuesto } \\
\text { como mínimo de los siguientes ambientes: dormitorio, baño, sala } \\
\text { de estar integrada con comedor y cocina equipada. Facilita la renta y } \\
\text { ocupación de estancias largas. Hostal: las habitaciones y los bańos de } \\
\text { aseo son compartidos, sin perjuicio de proporcionar otros servicios } \\
\text { complementarios. Deberá contar con un mínimo de } 5 \text { habitaciones. } \\
\text { Hostería: ofrece el servicio de hospedaje en habitaciones o cabañas } \\
\text { privadas, con cuarto de bańo y aseo privado; presta el servicio de } \\
\text { alimentos y bebidas. Deberá contar mínimo con } 5 \text { habitaciones } \\
\text { (Ministerio de Turismo, 2016, art. 12). }\end{array}$ \\
\hline
\end{tabular}

Fuente: elaboración propia a partir de la bibliografía consultada. 
En el Ecuador el sistema hotelero se reconoce por la categorización efectuada por el Ministerio de Turismo, de donde surgen: (1) hotel - de dos estrellas a cinco estrellas; (2) hostal de una estrella a tres estrellas; (3) hostería - hacienda turística - logde de tres estrellas a cinco estrellas; (4) resort - de cuatro estrellas a cinco estrellas; (5) refugio campamento turístico y casa de huéspedes es de categoría única (Ministerio de Turismo, 2016) (tabla 2).

Tabla 2. Categoría de los hoteles en Ecuador

\begin{tabular}{|c|c|}
\hline $\begin{array}{c}\text { Clasificación del establecimiento } \\
\text { de alojamiento turístico }\end{array}$ & Categorías asignadas \\
\hline Hotel & 2 estrellas a 5 estrellas \\
\hline Hostal & 1 estrella a 3 estrellas \\
\hline Hostería-Hacienda Turística-Lodge & 3 estrellas a 5 estrellas \\
\hline Resort & 4 estrellas a 5 estrellas \\
\hline Refugio & Categoría única \\
\hline Campamento turístico & Categoría única \\
\hline Casa de huéspedes & Categoría única \\
\hline
\end{tabular}

Fuente: Ministerio de Turismo, 2016.

Dentro de la provincia de Tungurahua se definen cuatro categorías: (1) una estrella o cuarta con siete establecimientos registrados; (2) dos estrellas o tercera con veintiséis instituciones; (3) tres estrellas o segunda con diez establecimientos; y (4) cuatro estrellas o primera con diez instalaciones. Los alojamientos más frecuentes en Tungurahua están entre dos y tres estrellas (Ministerio de Turismo, 2016) (tabla 3).

Tabla 3. Categorías de los hoteles en Tungurahua

\begin{tabular}{|c|c|c|c|c|c|c|}
\hline Categoría & Registro & Habita... & Camas & $\begin{array}{c}\text { Plaza } \\
\text { Camas }\end{array}$ & Mesas & $\begin{array}{c}\text { Plaza } \\
\text { Mesas }\end{array}$ \\
\hline I ESTRELLA O CUARTA & 7 & 106 & 192 & 221 & & \\
\hline 2 ESTRELLAS O TERCERA & 26 & 558 & 972 & 1161 & & \\
\hline 3 ESTRELLAS O SEGUNDA & 10 & 203 & 345 & 455 & & \\
\hline 4 ESTRELLAS O PRIMERA & 10 & 348 & 625 & 715 & & \\
\hline Total general & 53 & 1215 & 2134 & 2552 & & \\
\hline
\end{tabular}

Fuente: Ministerio de Turismo, 2016. 
En lo referente a los alojamientos en el Ecuador, el Ministerio de Turismo presenta estadísticas según la clase y por provincias, para el estudio de esta investigación se revisa la información presentada sobre la provincia de Tungurahua y su capital, la ciudad de Ambato (Ministerio de Turismo, 2016) (tabla 4).

Tabla 4. Alojamientos del Ecuador

\begin{tabular}{|l|c|c|c|c|c|c}
\hline \multicolumn{1}{|c|}{ PROVINGA } & Registrados & Habitaciones & Camas & Plazas camas & Mesas & $\begin{array}{c}\text { Plazas } \\
\text { Mesas }\end{array}$ \\
\hline AZUAY & 264 & 4.841 & 8.838 & 9.822 & & \\
\hline BOUVAR & 27 & 521 & 1.006 & 1.238 & & \\
\hline CANNAR & 35 & 738 & 1.213 & 1.338 & & \\
\hline CARCHI & 26 & 665 & 1.432 & 1.498 & & \\
\hline CHIMBORAZO & 88 & 1.791 & 3.754 & 3.929 & & \\
\hline COTOPAXI & 99 & 1.640 & 3.153 & 3.547 & & \\
\hline ELORO & 102 & 2.570 & 4.181 & 4.767 & & \\
\hline ESMERALDAS & 136 & 3.618 & 9.211 & 11.400 & & \\
\hline GALAPAGOS & 317 & 3.253 & 7.357 & 7.592 & & \\
\hline GUAYAS & 188 & 7.111 & 11.855 & 15.437 & & \\
\hline IMBABURA & 137 & 2.867 & 5.803 & 6.736 & & \\
\hline LOJA & 113 & 2.400 & 4.111 & 4.485 & & \\
\hline LOS RIOS & 41 & 920 & 1.651 & 1.984 & & \\
\hline MANABI & 305 & 5.801 & 12.014 & 16.134 & & \\
\hline MORONA SANTIAGO & 55 & 1.026 & 1.841 & 2.125 & & \\
\hline NAPO & 121 & 2.050 & 4.471 & 5.032 & & \\
\hline ORELLANA & 64 & 1.664 & 2.670 & 3.154 & & \\
\hline PASTAZA & 67 & 1.298 & 2.777 & 3.113 & & \\
\hline PICHINOHA & 769 & 14.983 & 23.367 & 32.416 & & \\
\hline SANTA ELENA & 269 & 5.145 & 12.073 & 15.326 & & \\
\hline SANTO DOMINGO & 106 & 2.591 & 4.594 & 4.736 & & \\
\hline SUCUMBIOS & 93 & 2.303 & 3.742 & 4.153 & & \\
\hline ZAMORA CHINCHIPE & 34 & 525 & 906 & 970 & & \\
\hline TUNGURAHUA & 202 & 3.850 & 7.821 & 9.479 & & \\
\hline
\end{tabular}

Fuente: Ministerio de Turismo, 2016.

Dentro de la Provincia de Tungurahua se han creado alojamientos en un número mayor para satisfacer la demanda creciente, en esta provincia existen 202 establecimientos habitaciones a nivel provincial y 3650; en la ciudad de Ambato los alojamientos que se disponen se reconocen: (1) hostal con 19 instalaciones registradas, (2) hostería con tres establecimientos y hoteles con 31 instituciones registradas (Ministerio de Turismo, 2016) (tabla 5).

En consecuencia, una vez analizadas las aristas de investigación es necesario realizar la evaluación de la situación financiera y el control de costos en empresas hoteleras mediante la categoría de análisis y revisión documental, que permitan identificar la fragmentación de la bibliografía y el estado de la práctica. 
Tabla 5. Clasificación de alojamientos de los hoteles en Ambato

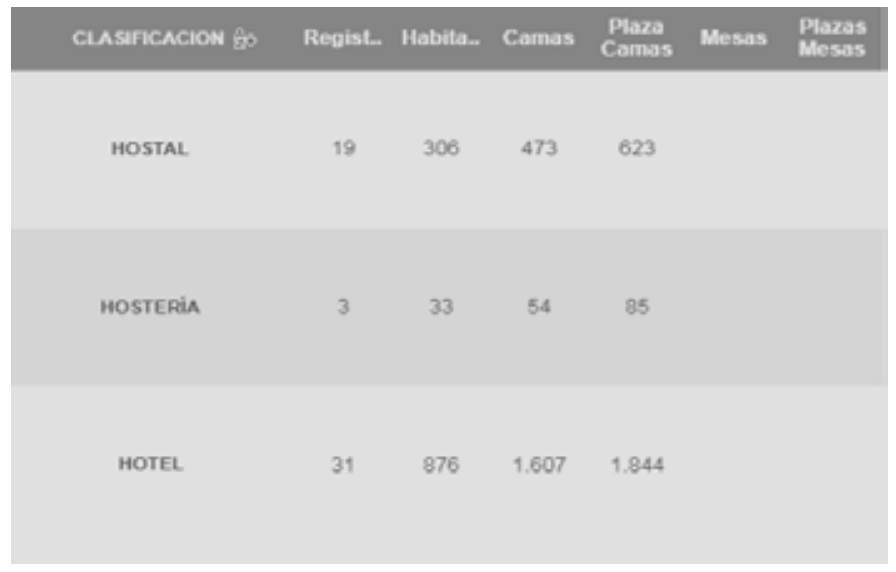

Fuente: Ministerio de Turismo, 2016.

\section{Materiales y MÉtodos}

La revisión documental constituye un elemento motivador para la realización de procesos investigativos de los estudiantes mediante el estudio de documentos a través de la identidad, elección y organización para incrementar y ahondar el tema abordado. A su vez, se caracteriza por su rigor metodológico, que dirige el camino a seguir en el transcurso investigativo, integrando un trabajo donde es necesario examinar antecedentes llevando a cabo inferencias y relaciones de ese saber acumulado ampliando más allá de lo estimado asumiendo un conjunto de procedimientos y técnicas de carácter explicativo, y su preeminencia es especificar, estudiar, interpretar y esquematizar el significado de hechos que se originan de manera natural; es así que la revisión documental se inscribe en el paradigma de investigación interpretativo, en la perspectiva de profundizar entre los diversos motivos de los hechos (Calcetero, 2018).

Para el diagnóstico de las variables se realizó una revisión documental de las disposiciones emitidas por los organismos de control, boletines, guías, lineamientos, para proceder a la triangulación de la información e investigación (tabla 6).

El Instituto Nacional de Estadísticas y Censos (INEC) presenta resultados al cerrar el ańo 2014, con respecto a los hoteles, restaurantes y servicios en el área de producción bruta, dividido en regiones y provincias, mostrándose en la provincia de la Sierra y Costa con cantidades más altas que en la región Insular y Amazónica, el análisis se lo efectúa a la producción de artículos para la venta, venta de artículos sin transformación, ingresos por servicios, otros ingresos por servicios, variación de existencias artículos para la venta sin transformación, compras netas de mercaderías y construcción, fabricación de activos con personal propio de la empresa (figura 1). 
Tabla 6. Revisión documental de control de costos hoteleros

\begin{tabular}{|c|c|c|c|}
\hline Documento & $\begin{array}{c}\text { Organismos } \\
\text { de control }\end{array}$ & Enfoque & Análisis categorial \\
\hline $\begin{array}{c}\text { Boletín de } \\
\text { septiembre 1. } \\
\text { de 2019 }\end{array}$ & SRI & $\begin{array}{c}\text { Servicios artísticos } \\
\text { y culturales }\end{array}$ & $\begin{array}{c}\text { Servicios artísticos y } \\
\text { culturales con tarifa 0\% }\end{array}$ \\
\hline $\begin{array}{c}\text { Boletín de } \\
\text { agosto de 2019 }\end{array}$ & $\begin{array}{c}\text { Ministerio de } \\
\text { Turismo }\end{array}$ & $\begin{array}{c}\text { Indicadores de medición } \\
\text { económica del turismo }\end{array}$ & $\begin{array}{c}\text { Ingresos y egre- } \\
\text { sos de divisas } \\
\text { Índices de precios } \\
\text { al consumidor }\end{array}$ \\
\hline $\begin{array}{c}\text { Boletín de } \\
\text { septiembre de 2019 }\end{array}$ & $\begin{array}{c}\text { Código Orgánico } \\
\text { de Producción }\end{array}$ & $\begin{array}{c}\text { Implementación del nuevo } \\
\text { formulario autorizado por } \\
\text { importaciones de con- } \\
\text { sumo a medicamentos }\end{array}$ & $\begin{array}{c}\text { Instructivo soporte de } \\
\text { uso obligatorio para la } \\
\text { adquisición a consumo } \\
\text { de las partidas tributarias } \\
\text { incluidas como produc- } \\
\text { tos farmacéuticos del } \\
\text { Arancel del Ecuador }\end{array}$ \\
\hline
\end{tabular}

Fuente: elaboración propia a partir de la bibliografía consultada.

Figura 1. Producción bruta y total según regiones y provincias

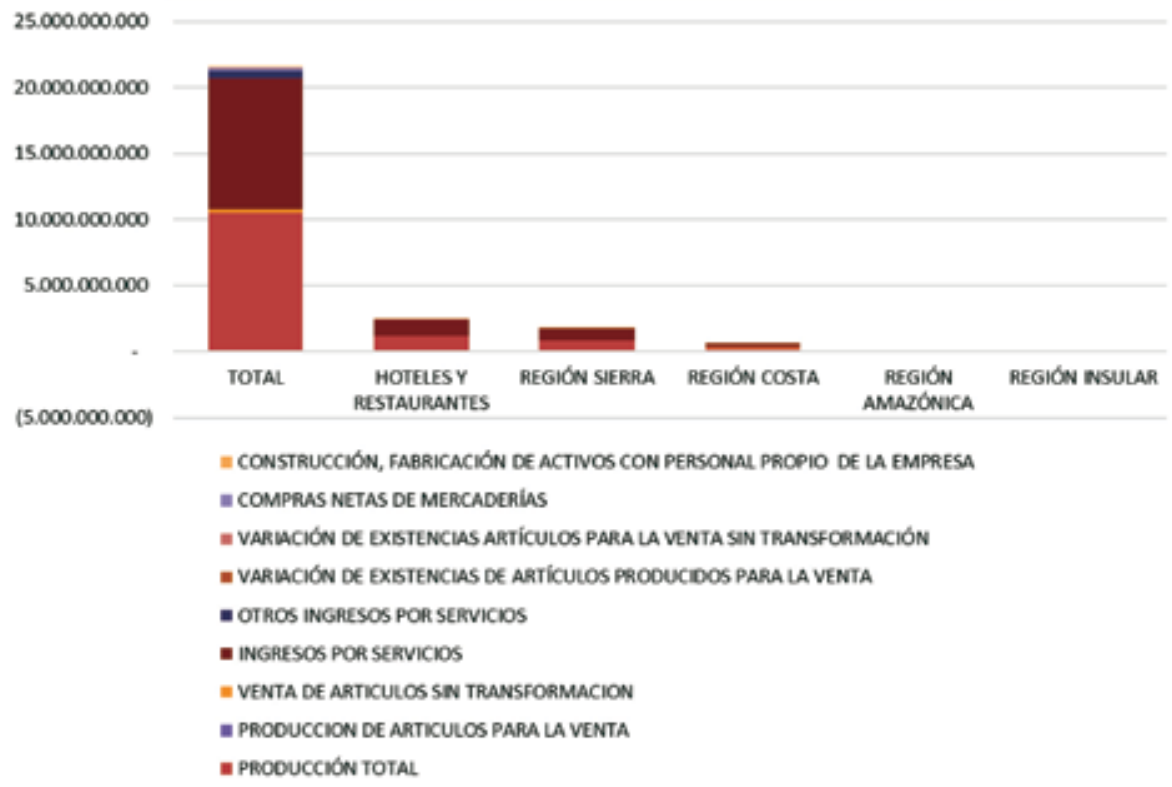

Fuente: INEC, 2014. 
En la provincia de Tungurahua se refleja que hay ingresos por servicios de 7.727.235, lo cual quiere decir que los otros elementos que integran la producción total de los hoteles y servicios presentan un $0 \%$, como lo es la venta de artículos sin transformación, otros ingresos por servicios, variación de existencias de artículos producidos para la venta, variación de existencias de artículos sin transformación, compras netas de mercaderías y contribución fabricación de activos con personal propio de la empresa (INEC, 2014) (figura 2).

Figura 2. Producción total en la provincia de Tungurahua

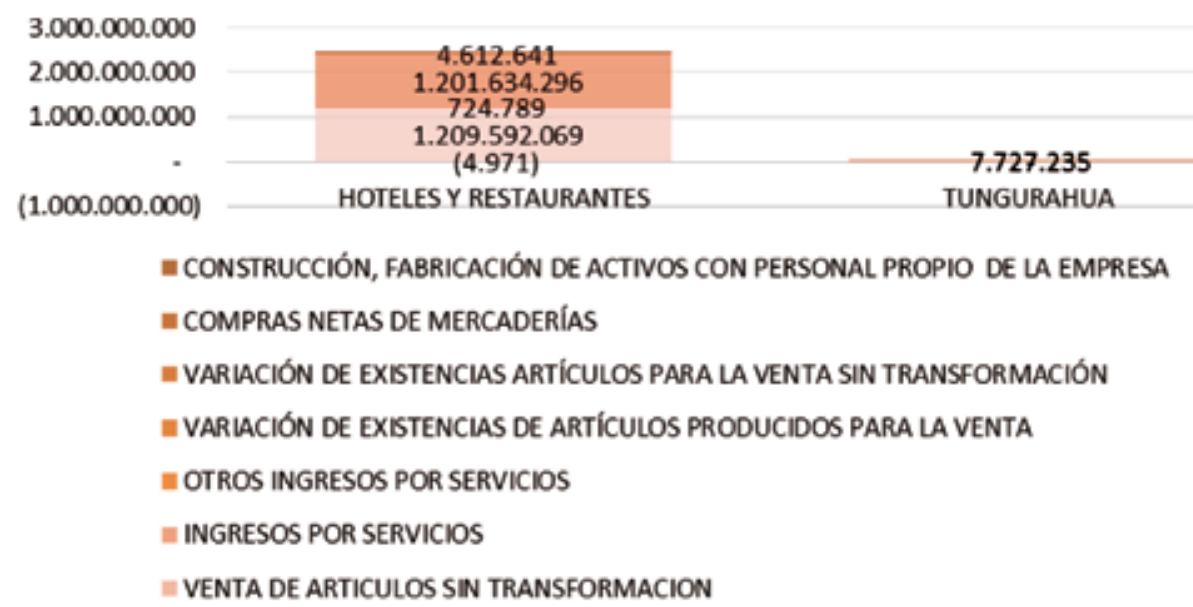

Fuente: INEC, 2014.

Se plasma que en los reportes que informa el Instituto Nacional de Estadísticas y Censos no emiten las herramientas que se requieren para estimar y verificar los indicadores que forma la evaluación financiera para analizar si los hoteles en Tungurahua están obteniendo como resultado rentabilidad o disminución de dinero (figura 3).

A partir de la bibliografía consultada y analizados los resultados del análisis documental, es necesario reflexionar sobre uno de los componentes del proceso estratégico, denominado "soporte estratégico", que dentro de sus elementos comprende el control, que permite la fluidez dentro de los sistemas de control y comunicación de las organizaciones, y en consecuencia, todo dependerá del estilo de la dirección, la recompensa de incentivos, el clima, la cultura y el liderazgo. Por lo tanto, se pueden inferir los factores que intervendrán dentro de los valores compartidos en las organizaciones que brindan un servicio (Mayorga \& Llagua, 2018). 
Figura 3. Gastos de operación, intereses

e impuestos indirectos según provincias

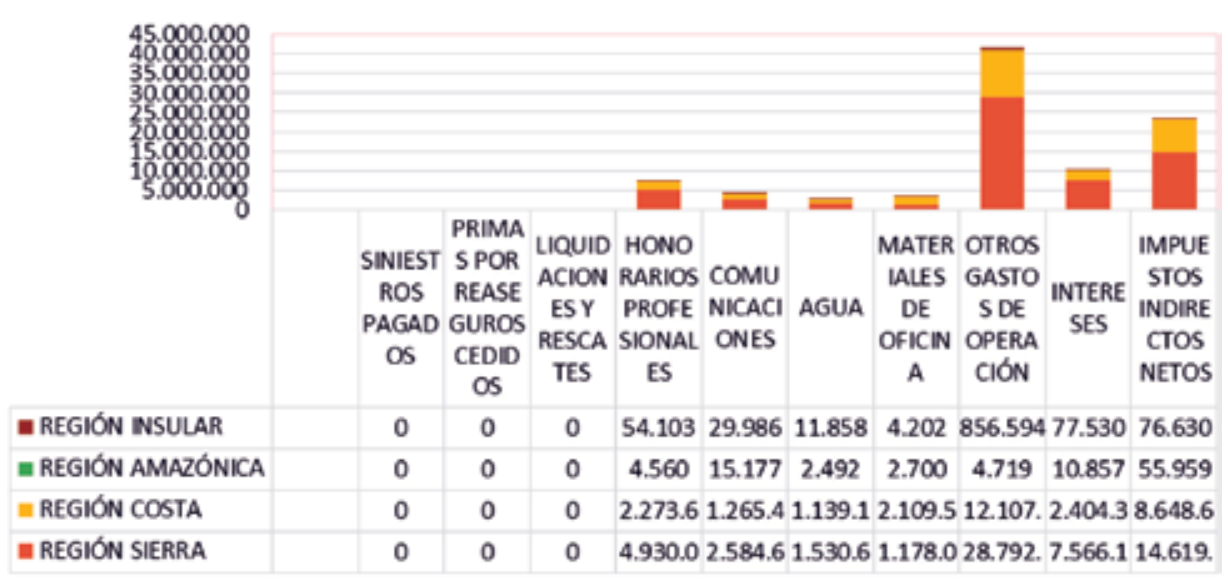

Fuente: INEC, 2014.

\section{Resultados Y Discusión}

Para esta investigación se ha procedido a realizar una revisión documental de los organismos de control que rigen a las organizaciones hoteleras para verificar si estas entidades han realizado boletines, guías o lineamientos sobre el control de los costos de producción en los hoteles; de cara a la aplicación de estas técnicas de investigación se puede determinar que los organismos de control y las organizaciones hoteleras se enfocan vagamente en la rendimiento de servicios acorde a las perspectiva del cliente, y se ofrece una escasa información sobre la organización de costos de producción de las empresas hoteleras, los organismos de control se basan en otras áreas como servicios artísticos y culturales, indicadores de medición económica del turismo, e implementación del nuevo formulario autorizado por la Agencia Nacional de Regulación, Control y Vigilancia Sanitaria para Importaciones a Consumo de Medicamentos, lo cual no permite fortalecer aspectos importantes como los costos de producción e indicadores de evaluación financiera de las respectivas organizaciones estudiadas.

Ruiz, González, \& Alba (2018) sustentan que el análisis del comportamiento organizacional permite la identificación de falencias ante las situaciones que se presentan en el desarrollo de la operación de la compañía, de forma que se realicen modificaciones pertinentes en la manera de actuar a nivel individual, grupal y organizacional, y se tengan en cuenta en el desarrollo de la operación del negocio y en la implementación de nuevos proyectos.

En tal sentido, es necesario plasmar un modelo conceptual elaborado como base para la representación de los componentes y las relaciones que se presentan para un fenómeno (Galarza, 2007), (Ferriol, 2011), (Mayorga, 2018) en donde se 
reconocen como aspectos referidos al diseño del modelo, los que tratan Galarza (2007), Ferriol (2011) y Mayorga (2018).

1. Los componentes del modelo se definen como las variables fundamentales: herramientas de gestión y evaluación financiera.

2. Los elementos, que se definen como las dimensiones de las variables: 1 . medición de la evaluación financiera: indicadores financieros, indicadores económicos, análisis financieros, estados financieros.

3. Las "fronteras", que establecen los términos y límites en que actúa la organización estudiada, los que se consideran apoyo para el desarrollo de la concepción de integración planteada:

a. Marco jurídico vigente.

b. Organismo rector de control y fiscalización, se reconoce a la Ley de Régimen Tributario Interno (LORTI) SRI Ley de Turismo que a través de la unidad de fiscalización viabiliza la aplicación de procedimientos coherentes con la necesidad nacional.

c. El resultado de las interacciones del modelo propuesto constituye el informe de mejora continua de resultados.

Figura 4. Modelo conceptual de gestión de costos y evaluación financiera

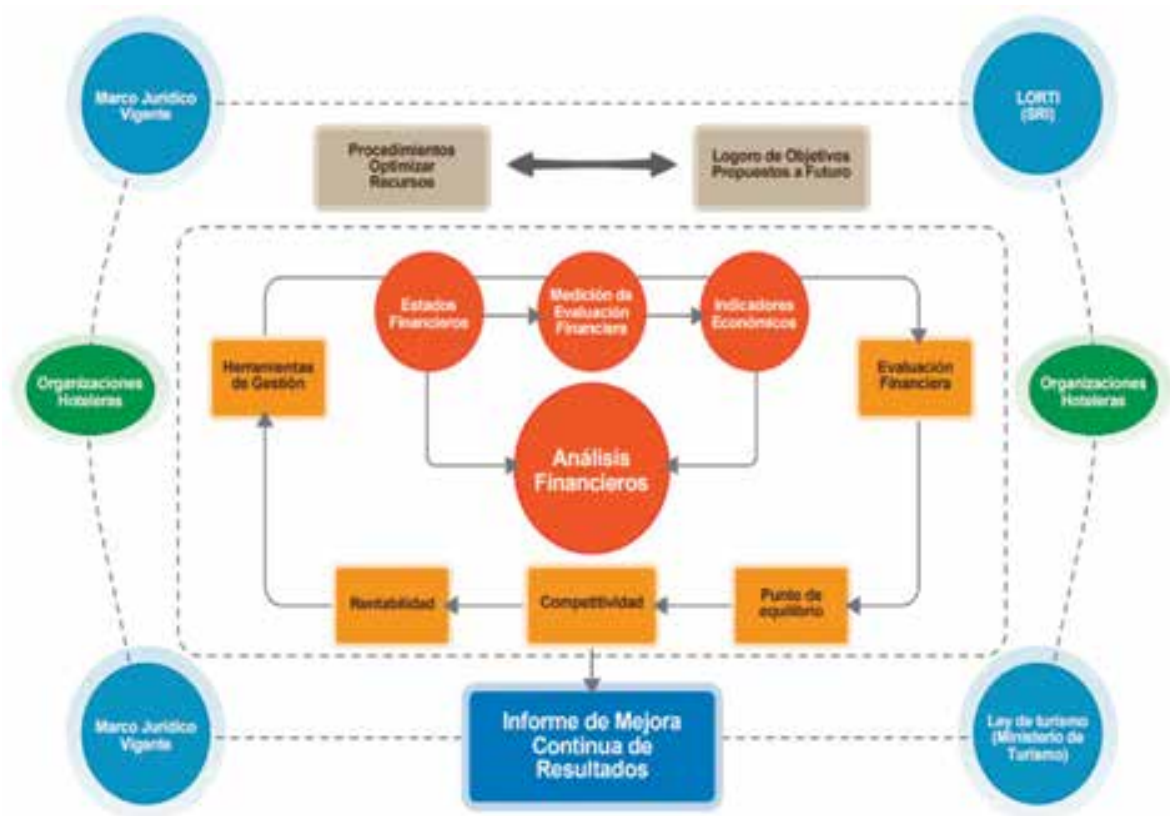

Fuente: elaboración propia. 


\section{Conclusiones}

El control de costos de producción mediante la adopción de herramientas de gestión de costos permite la optimización de recursos con acciones estratégicas para la toma de decisiones con el objetivo de lograr objetivos y detectar dificultades futuras de la situación actual de la organización, con la finalidad de determinar procesos de manera eficiente a través del seguimiento de las actividades, reducción y mejora continua de la estructura de costos que garantiza el éxito y el control de las mismas.

Las perspectivas de las organizaciones del sector hotelero deben enfocarse a seguir un modelo para la toma de decisiones con vistas a mejorar su sistema de control de costo y su rentabilidad, con lo que se pueda lograr en un futuro una verdadera rentabilidad enfocada a cumplir con las expectativas de los clientes (turistas), mejorar la calidad de existencia de los clientes internos y de la sociedad misma.

\section{REFERENCIAS BIBLIOGRÁFICAS}

Aponte, M. R. (2017). La evaluación financiera de proyectos y su aporte en la generación de valor corporativo Ciencia y Poder. Revista Ciencia y poder Aéreo, 12 (1), 144-155. doi:http://dx.doi.org/10.18667/cienciaypoderaereo.567.

Asamblea Nacional. (29 de diciembre de 2010). Artículo 62 [Tíulo II] Preliminar del Objetivo y Ámbito de Aplicación Acceso a la Banca Pública [Registro Oficial 351 de 2010].

Asamblea Nacional. (17 de diciembre de 2013) Artículo 13 [Título III] Ley de Régimen Tributario Interno [Registro Oficial 145 de 2013].

Aspiolea, A. M. (2017). Modelo de gestión estratégica de costo para instalaciones. Revista Especial Única, 6, 437-447. Obtenido de http://revistas.unica.cu/index.php/uciencia/ article/viewFile/963/815.

Calcetero, G. J. (2018). Una revisión a la dimensión ambiental y al desarrollo de capacidades humanas. Tabula Rasa, 17, 385-407. Obtenido de http://www.scielo.org.co/ pdf/tara/n28/1794-2489-tara-28-00385.pdf.

Calvo, L. J. (2010). Componente finanzas y su evolución. Revista Apuntes Contables (14), 43-102. Obtenido de https://revistas.uexternado.edu.co/index.php/contad/article/ view/1663/1501.

Cárdenas, R. A. (2016). En Costos II. La gestión gerencial IMPC. México: IMPC.

Católico, S. D. (2019). La incidencia de las normas internacionales de la infomación financiera sobre la revelacion de la informacion contable en empresas colombianas. Revista Apuntes Contables (23), 85-105. doi:https://doi.org/10.18601/16577175.n23.06Diego.

Constitución de la República. (22 de enero de 2015). Artículo 23 [título II] del procedimiento para la prestación de servicios materiales a través de la iniciativa privada [Registro Oficial 244 de 2015].

Ediciones, E. (2016). En Costos y gestión empresarial. Ecoe. 
Ferriol (2011). Los modelos de gestión institucional en el contecto educativo actual y su articulación en las escuelas de idiomas. Órbitra Científica. Obtenido de http://revistas. ucpejv.edu.cu/index.php/rOrb/article/download/723/998.

Galarza, M. (2007). Modelo integracionista de las variables fundamentales que influyen sobre la eficiencia acádemica en instituciones de educación superior. XVII (1 2012). Recuperado el 20 de abril de 2019, de file://C:/Users/Mary/Downloads/5-18-1PB\%20(1).pdf.

García, A. M. (2016). Gestión logística integral y las mejores prácticas en la cadena de abastecimiento (2. ${ }^{\mathrm{a}}$ ed.). Colombia: Ecoe.

Gómez, O. E. (2018). Gestión estratégica de costos: una herramienta de competitividad. Revista Espacios, 39(32), 39. Obtenido de https://www.revistaespacios.com/a18v39 n32/18393204.html.

González, R. C. (2016). Análisis de la problemática de la gestión de proyectos estudio en el contexto empresarial. Revista Ciencias Estratégicas, 24 (35), 119-133. doi:rces.v24n35.a7.

INEC. (15 de septiembre de 2014). Hoteles, restaurantes y servicios. Obtenido de https:// www.ecuadorencifras.gob.ec/hoteles-restaurantes-y-servicios-2014/.

Jiménez, V. L. (2016). Los costos de mala calidad como quinto elemento del costo. Revista Facultad de ciencias económicas investigación y reflexión, 24(1), 63-84. Obtenido de https://www.redalyc.org/pdf/909/90943602006.pdf.

Liendo Villarreal, J. C. (2016). Los costos basados en actividades (abc) y su influencia en la rentabilidad del gran hotel alturas de la ciudad de Huaraz, periodo 2014-2015.

Mayenberger, A. S. (2017). Evaluación operacional y financiera aplicada a la Industria Hotelera. Colombia: Universidad Externado de Colombia.

Mayorga-et al., (2018). Conceptual model and process applied in management auditic with focus in social responsibility through open source tools. In 2018 13th Iberian Conference on Information Systems and Technologies. IEEE.

Mayorga, M., \& Llagua, V. (2018). La evaluación del sistema del control interno como soporte estratégico en la gestión de objetivos en las finanzas populares del Ecuador. Apuntes Contables, 117-126.

Ministerio de Turismo. (2016). Turismo en cifras. Recuperado el 25 de septiembre de 2019, de https://www.turismo.gob.ec.

Ministerio de Turismo. (18 de febrero de 2016). Reglamento de Alojamiento Turístico [Registro Oficial 465 de 2016].

Otálora, B. J. (2016). Sistemas de gestión de costos en las cooperativas de ahorro y crédito de Barranquilla. Revista Cuadernos de Contabilidad, 17 (44), 349-375. Obtenido de http://www.scielo.org.co/pdf/cuco/v17n44/v17n44a02.pdf.

Pereira, B. C. (2019). Actualidad en la gestión empresarial en las pymes. Revista Apuntes Contables (24), 7. doi:10.18601/16577175.

Rodríguez Benavides, L. G. (2017). La asignación de costos y la rentabilidad en el hotel Volcano de la ciudad Baños de agua Santa (trabajo de grado), Universidad Técnica de Ambato, Ambato. Obtenido de http://repo.uta.edu.ec/bitstream/123456789/25907/1/ T4045i.pdf. 
Rodríguez Flores, G. J. (2018). Factores claves del servicio para la instalación del Ecolodge de Intijecanan Distrito de Pariahuanca (trabajo de grado), Universidad Nacional Santiago Antúnez de Mayolo, Perú. Obtenido de http://repositorio.unasam.edu.pe/bitstream/ handle/UNASAM/2362/T033_45731997_T.pdf?sequence $=1$ \&isAllowed $=y$.

Rodríguez, López., M., Rodríguez, Samiego, J. (2018). Particularidades del costo en las Universidades. Revista Apuntes Contables (21), 8. doi:https://doi.org/10.18601/16577175. n21.08.

Roque, I. D. (2019). Como vincular la información que brinda la contabilidad de gestión ambiental con los proyectos de inversión. Revista Apuntes Contables (23), 11. doi:https:// doi.org/10.18601/16577175.n23.02Daniel Isaac Roque1 marlene Cañizares Roig2.

Ruiz, C., González, V., \& Alba, M. (2018). El comportamiento organizacional y las Normas Internacionales de Información Financiera: experiencia en una pyme. Apuntes Contables, 57-72.

Serrano, P. G. (2016). Diseño de proyectos sociales, aplicaciones prácticas para su planificación, gestión y evaluación. Revista Complutense de Educación, 28(2), 267. doi:666 Rev. complut educ. 28 (2) 2017: 663-671 RESEÑAs http://dx.doi.org/10.5209/RCED.5262.

Villanueva, A. L. (2015). Gobernanza y gestión pública. México: Fondo de Cultura Económica. 